\title{
Laplace-Based Predictive Estimation of Loss-of-Control Boundaries on a Transport Aircraft
}

\author{
Kiran Rajaram*, Melvin Rafi, \\ Faculty: James E. Steck, Animesh Chakravarthy \\ Department of Aerospace Engineering, College of Engineering
}

Loss-of-control events are the greatest contributing factor to accidents amongst commercial aircraft. In this research, an approach to predicting the onset of loss-of-control events is developed using a Laplace-based method, where a state space model is used to simulate the dynamics of the aircraft. Laplace transformations are then used to calculate the control authority required to reach a predefined limit on the aircraft's angle-of-attack, where the limit represents the control loss boundary. This method is applied to the short period dynamics of the NASA Generic Transport Model, and the remaining amount of allowable control authority the pilot has before reaching a loss-of-control situation is then determined. This boundary is graphically presented through a three-dimensional display. Simulations are run within MATLAB/Simulink ${ }^{\circledR}$, and results demonstrate that the system is able to successfully predict the aircraft's proximity to a control loss situation. 